\title{
La práctica pre profesional en el desarrollo de habilidades profesionales
}

\section{Pre professional practice in the development of professional skills}

*Melquiades Mendoza Pérez ${ }^{1}$, Gina Silvana Venegas Álvarez², Alberto Rodríguez Rodríguez ${ }^{3}$

${ }^{1}$ Universidad Técnica de Cotopaxi, Facultad de Ciencias Humanas y de la Educación, Escuela de Comunicación Social. Av. Simón Rodríguez s/n Barrio El Ejido Sector San Felipe, provincia Cotopaxi. EC.05-01491.

Latacunga, Ecuador.*Melquiades.mendoza@utc.edu.ec

${ }^{2}$ Universidad Técnica de Cotopaxi, Facultad de Ciencias Humanas y de la Educación, Escuela de Comunicación Social. Av. Simón Rodríguez s/n Barrio El Ejido Sector San Felipe, provincia Cotopaxi. EC.05-01491. Latacunga, Ecuador.gina.venegas@utc.edu.ec

${ }^{3}$ Universidad Estatal del Sur de Manabí. Facultad de Ciencias de la Ingeniería, Escuela en Sistemas Computacionales. Manabí, Ecuador. Km 1 1² vía Noboa s/n Campus Los Ángeles. Jipijapa- Ecuador, drrodriguezcc9564@gmail.com

Rec.: 17.02.2016. Acept.: 29.04.2016. Publicado el 30 de diciembre de 2016 Rev. Cienc. Soc. y Econ. UTEQ (2016), 1(1): 83-92

\section{Resumen}

El presente trabajo está referido al tratamiento que debe dársele al desarrollo de las habilidades profesionales de los futuros graduados durante su formación. Los resultados se obtienen a partir de la utilización de métodos teóricos como el analítico-sintético, inductivo-deductivo, el enfoque de sistema. Las particularidades y las características del trabajo en el desarrollo de habilidades profesionales revelan la necesidad de profundizar en los conocimientos pedagógicos y en las características socioculturales y económicas de los contextos. Para la solución de la problemática abordada se propone un sistema de actividades que involucra a la universidad y la actuación conjunta y coordinada de todos los actores que participan del proceso.

Palabras clave: habilidades profesionales, desarrollo de habilidades, sistema de actividades. 


\begin{abstract}
This paper refers to the treatment to be given to the development of professional skills in future graduates. The results were obtained through the use of such theoretical methods as analyticalsynthetic, inductive-deductive, and system approach. The characteristics of the work in the development of professional skills reveal the need to increase pedagogical knowledge as well as awareness of socio-cultural and economic characteristics of the contexts. To solve this problem, a system of activities including the university and the coordinated action of all the actors involved in the process is proposed.
\end{abstract}

Key words: professional skills, skills development, system of activities.

\title{
Introducción
}

La educación ecuatoriana, a partir de las necesidades sociales, debe fomentar el nivel de motivación de los estudiantes por el trabajo, crear en ellos una conciencia de productores en contra de la corriente contemporánea del consumismo que se mueve por muchos países (García, 2006).

Para lograr el encargo que la sociedad le da a la universidad es necesario vincular el proceso formativo con la vida, relacionarlo con el proceso social y productivo del contexto donde está ubicada la universidad, llevar y discutir en el aula los problemas que se presentan en la comunidad, en el contexto micro, meso y macro, y buscar soluciones a partir del aprendizaje y aplicación de los contenidos de las diferentes asignaturas (Hernández \& Maquilón, 2010).

Entre motivos e intereses existe una estrecha relación y el interés se manifiesta como un motivo especifico de la actividad cognoscitiva, constituyendo una orientación específica de la personalidad. Este análisis es comprensible al conocer la correspondencia de lo cognitivo y lo afectivo como aspectos condicionantes (Blas, 2007). 
González (1989) dirige la atención hacia un conjunto de conocimientos, habilidades, capacidades, motivos e intereses profesionales, que juegan un papel decisivo en el desarrollo de la autovaloración del sujeto y de cualidades de la personalidad. El proceso comienza en edades tempranas y continúa después de la inserción del joven en el ejercicio de la profesión y lo periodiza en cuatro etapas esenciales: de formación vocacional general, donde se manifiesta en edades tempranas, primero como intereses cognoscitivos y después como intereses profesionales. De preparación para la selección profesional que es dirigida a desarrollar los intereses cognoscitivos, conocimientos y habilidades relacionadas con las asignaturas y demás actividades del proceso formativo. $\mathrm{Su}$ objetivo es preparar al estudiante para la selección profesional auto determinado y consciente. De formación y desarrollo de intereses y habilidades profesionales, donde coincide con la entrada del joven a un centro de formación profesional (superior o medio). Tiene como finalidad desarrollar valores, convicciones, intereses y conocimientos hacia el contenido de la profesión, así como la asimilación de habilidades y hábitos para configurar su identidad profesional con vista desempeño. De consolidación de los intereses, conocimientos y habilidades profesionales, no se vincula necesariamente al egreso del centro de formación. El joven debe dominar conocimientos, habilidades y destrezas para perfeccionarlas y manifestarlas en el resto de la vida y reafirmar los intereses profesionales.

Para alcanzar estos propósitos se debe garantizar un trabajo coherente que se inicie desde los primeros ciclos, aprovechando todas las posibilidades que brinda el proceso formativo para trasmitir a los estudiantes el amor hacia la profesión por la cual siente vocación y un sólido trabajo metodológico que lleve consigo el compromiso de servir a la sociedad (Polanco, 2005).

No obstante al trabajo que se desarrolla en la universidad en función del desarrollo de la orientación profesional, aún existen insuficiencias, en lo fundamental en el trabajo que se realiza como universidad en la atención al profesional en formación cuando realizan su práctica pre profesional para desarrollar en ellos habilidades profesionales. Por lo anteriormente planteado se formula el siguiente problema científico: ¿Cómo desarrollar habilidades profesionales en el profesional en formación de la Universidad Técnica de Cotopaxi? 
Mendoza et al., 2016

Para dar solución al problema se formula el siguiente objetivo, elaboración de un sistema de actividades por los profesores en formación en la práctica pre profesional e investigativa, liderada por el profesor tutor de la universidad

\section{Metodología}

El trabajo de formación vocacional y orientación profesional en los centros docentes, se desarrolló por medio de las diferentes asignaturas que componen el plan de estudio y mediante un sistema de actividades formativas en estrecha relación con el contexto, entre las cuales ocupan un lugar significativo casas de cultura, museos, bibliotecas, instituciones laborales, centros de investigación, laboratorios, círculos de interés científico - técnico, conferencias, exposiciones, divulgación profesional y otras (Aneas, 2003).

Según, Del Pino y Recarey (2005), la orientación significa ayuda y no imposición del punto de vista de una persona sobre otra, no es tomar decisiones por alguien, sino ayudarlo a resolver los problemas, a desarrollar sus puntos de vista y a responsabilizarse con sus propias decisiones. Se ayudó al docente a conocerse y mejorarse a sí mismo, en relación con el medio en que se desenvuelve, guiando al estudiante para que movilice conscientemente sus posibilidades, enfrente sus limitaciones y decida el curso de su vida, de acuerdo a la técnica de González (1989).

Así, la orientación profesional (Castro, 1991) permitió juicios y valoraciones propias de la profesión, con una fuerte carga afectiva en el proceso de enseñanza aprendizaje. Donde el docente vinculó su experiencia, conocimientos, habilidades y valores adquiridos, en la interrelación con el alumno en el proceso formativo durante el tránsito por la universidad.

Para lograr la motivación y orientación profesional, fue indispensable fortalecer el modelo del profesional que se quiere obtener, como condición básica para el desarrollo de intereses cognoscitivos, conocimientos y habilidades específicas, relacionadas con aquellas asignaturas en las cuales el docente muestra marcadas inclinaciones y posibilidades en el orden intelectual. 
En la universidad, el profesor es quien concreta los lineamientos trazados por la institución, en la medida para dar cumplimiento a los planes de estudio, indicaciones metodológicas y documentos normativos. En este sentido, el profesor debe ser un activista de la política universitaria, un defensor. Debe ser un ejemplo, comenzando por el requisito de ser un buen profesor, un trabajador disciplinado y un profesional con espíritu de superación. Así mismo, la ejemplaridad se demuestra en la puntualidad, disciplina, calidad de las clases, cumplimiento de las normas, en las relaciones con los alumnos y compañeros profesores y en la exigencia para consigo mismo y para con los demás. Por estas razones, en la formación vocacional, cada actividad que se realiza posee una relación particular con el desarrollo del estudiante, que incluso varía de acuerdo, no solo con los estadios por los que pasa su vida, sino con sus propias particularidades individuales y en el contexto social en el que se desarrolla.

La clase, en cualquiera de sus formas se propició como vía principal para la motivación y orientación profesional, caracterizada por ser el espacio sistemático y conscientemente planificado donde el estudiante se encuentra con la cultura general a través de las diferentes asignaturas. Donde el profesor proyecta en el aula su comunicación y autorrealización, destacando la significación de la ciencia y aplicaciones que contiene. Es decir, el profesor precisó, desde el trabajo metodológico, todas las potencialidades de la asignatura para la orientación profesional y las vías para desplegarlas en cada clase, garantizando, desde el diagnóstico continuo de sus estudiantes, la problematización de su relación con su profesión.

En consecuencia, se emplearon métodos teóricos como el analítico-sintético, el inductivodeductivo y el enfoque de sistema, para el análisis y el procesamiento de los datos obtenidos durante la investigación a partir de considerar la formación vocacional y orientación profesional en los centros docentes, como un proceso que se desarrolla mediante un sistema de actividades formativas en estrecha relación con el contexto, en el que los profesionales juegan un papel significativo. Se apunta también a un análisis de otras perspectivas pedagógicas, como la elaboración de un sistema de actividades a desarrollar por los profesores en formación en la práctica pre profesional e investigativa, liderada por el profesor tutor de la universidad. 
Mendoza et al., 2016

\section{Resultados y discusión}

En su práctica el profesional en formación cumplió los objetivos que demanda la profesión que estudia, los cuales son:

1) Desarrollo personal y la transformación del contexto y desarrollo socioeconómico sostenible, cultural, educativo, turístico, ambiental, entre otros.

2) Contribuir a la solución de los problemas que afectan el desarrollo contextual, evidenciando disciplina, espíritu crítico y auto-crítico, conciencia de la necesidad de auto-superación constante.

3) Transferencia de tecnología y cultura a los contextos.

En el referido proceso se escogieron aquellas habilidades que tributen al modo de actuar propio del profesional que, de acuerdo con su objeto de trabajo, se concretó en el perfil de egreso. Pues las habilidades profesionales, en opinión de Márquez (2005), "Son aquellas que garantizan el éxito, en la ejecución de la actividad de la profesión, y la solución de los más diversos problemas de la especialidad".

Por otra parte, se considera que son "el contenido lógico de las acciones que realiza el profesional al interactuar con los objetos de la profesión" y considerando que a partir de la clasificación dada por Álvarez (2000) y Fuentes (1996), las habilidades se clasifican en tres grupos:

1) Habilidades específicas. Son habilidades propias de las ciencias de las profesiones o de las tecnologías que son objeto de estudio o trabajo, estas habilidades se llevan a las disciplinas y se concretan en los métodos de trabajo que deben aparecer como contenido de la práctica. 
2) Habilidades lógicas o intelectuales son aquellas que contribuyen a la asimilación del contenido de las disciplinas y que sustentan el pensamiento lógico, tanto en el aprendizaje como en la vida.

3) Las habilidades de comunicación propias del proceso docente que son imprescindibles para el desarrollo de este.

De acuerdo con este mismo autor en el proceso de aprendizaje las habilidades se van perfeccionando en dos direcciones y devienen habilidades de mayor complejidad. En la primera dirección, se produce un perfeccionamiento de la habilidad de manera consciente mediante el cumplimiento de acciones teóricas y prácticas cada vez más complejas. En la segunda se mantiene el grado de complejidad en los problemas a solucionar, pero se ejercitan hasta que se automaticen y el sujeto va siendo cada vez menos consciente de sus acciones hasta que se logra formar una habilidad automatizada o hábito.

En la práctica pre profesional la formación y desarrollo de habilidades técnico profesionales se logró, mediante la actuación conjunta y coordinada de todos los actores que participaron en el proceso.

Las habilidades son un componente esencial de la actividad humana, razón por la cual su formación y desarrollo está estrechamente vinculado a la práctica (Álvarez, 2000). Las habilidades profesionales adquieren una dimensión particular en el proceso de formación, pues significan el rol de la práctica pre profesional en consonancia con las necesidades de la operacionalización de la actividad profesional (Barquero, 1997).

\section{Conclusiones}

El estudio de los fundamentos teóricos y metodológicos de la problemática abordada, unido a los resultados del diagnóstico del estado del campo de acción de la investigación, permitió determinar que para la solución del problema científico presentado debía concebirse un sistema 
Mendoza et al., 2016

de actividades que responda al perfil de egreso del profesional e involucre a los factores, sectores y actores sociales en el proceso. En este contexto, la Universidad Técnica de Cotopaxi, logró un acercamiento a la solución de un problema social como lo es la formación del profesional.

\section{Referencia bibliográfica}

Álvarez, M. (2000). “La Educación avanzada, una alternativa para la preparación de los gestores de formación vocacional pedagógica.” Tesis de Maestría. Biblioteca Virtual CUJAE, Ciudad Habana, p. 10.

Álvarez, CM. (1992). La escuela en la vida. La Habana: Ed. Pueblo y Educación.

Aneas, A. (2003). Competencias profesionales. Análisis conceptual y aplicación profesional, conferencia para el Seminario Permanente de Orientación Profesional, organizado por el Departamento de Métodos, Investigación y diagnóstico en Educación de la Universidad de Barcelona.

Barquero, R. (1997). Vigotsky y el aprendizaje escolar. Buenos Aires, Argentina: Editorial Aique.

Blas, Francisco de Asís (2007): Competencias profesionales en la formación profesional. Madrid: Alianza.

Castro, PL. (1991). "El Sistema familiar en el proceso de la vocación de los hijos." Tesis de doctorado. Biblioteca MINED, La Habana. p. 24.

Del Pino. JL. (1988). “Motivación y orientación profesional en el ámbito educativo.” Ciudad de La Habana, Facultad de Ciencias de la Educación. s/p

Del Pino, JL. y Recarey, S. (2005). “Diagnóstico individual y grupal. Orientación y prevención en el contexto escolar.” Módulo II Segunda Parte. Maestría en Ciencias de la Educación. Editorial Pueblo y Educación. Ciudad Habana.

Fuentes, H. (1996). Dinámica del Proceso de Enseñanza - Aprendizaje. - Santiago de Cuba.

García, G. (2006). Compendio de pedagogía, Editorial Pueblo y Educación.

González, F. (1989). Motivación profesional en adolescentes y jóvenes. La Habana. Editorial Ciencias Sociales, p. 14-17

González, MV. (1989). Niveles de integración de la motivación profesional”. Tesis de doctorado en Ciencias Psicológicas. La Habana. 
González, RM. (2008). Competencias genéricas y formación profesional: un análisis desde la docencia universitaria», en Revista Iberoamericana de Educación, No 47. Madrid, España.

Márquez, A. (2005) Habilidades: Reflexiones y Proposiciones para su evaluación-conferencias. Santiago de Cuba.

Maquilón, \& Hernández (2011). Influencia de la motivación en el rendimiento académico de los estudiantes de formación profesional. Revista Electrónica Interuniversitaria de Formación del Profesorado, 14(1), 81-100. Asociación Universitaria de Formación del Profesorado, Zaragoza, España.

Polanco, A (2005). La motivación en los estudiantes universitarios. Revista Electrónica "Actualidades Investigativas en Educación", 5(2), 1-13 Universidad de Costa Rica, San Pedro de Montes de Oca, Costa Rica. 